\title{
A Fast Method of Calculating Diffraction Loss Between Two Facing Transducers
}

\author{
ABDULLAH ATALAR
}

\begin{abstract}
A fast method of calculating the diffraction loss between two facing circular ultrasonic transducers of unequal size is presented. This problem is directly applicable for minimization of diffraction loss in acoustic lens design. Graphs for amplitude and phase are presented that can be used to design lenses with the optimal transducer size for minimum diffraction loss. The theory is extended to include the diffraction loss determination in anisotropic materials. The results are in good agreement with previous experimental and theoretical results of the equal transducer size case. The effect of diffraction on pulsed excitation is also treated.
\end{abstract}

\section{IntRoduction}

A COUSTIC FIELDS generated by circular or rectangular shaped acoustic transducers in continuous as well as pulsed wave excitation cases have been obtained theoretically as well as experimentally. Zemanek [1] has presented the acoustic field patterns for a circular transducer in the near field for an isotropic propagation medium. He has calculated the pressure data by double numerical integration of the exact integral solution. Lockwood and Willette [2] have introduced a faster solution method for the same problem by use of the Fourier transform of the impulse response [3]. Beaver [4] investigated the nearfield pulse response of circular radiators. Harris [5] reviewed the theoretical approaches used in solving the problem. Several authors [6]-[8] have also considered the problem under slightly different conditions. A recent paper presents theoretical and experimental pressure fields of an axisymmetric transducer [9].

Diffraction loss for a transducer facing a planar reflector as a function of the distance between the reflector and the transducer is of considerable interest [10] and it is thoroughly investigated. This problem is especially important in pulse-echo attenuation measurements. Papadakis [11]-[13] generalized the diffraction loss calculation to anisotropic materials by introducing correction terms as given by Waterman [16]. Gitis and Khimunin [15] gave a review of the work up to that time. The diffraction loss generally increases as the distance between the transmitter and receiver increases. The loss is zero when the distance is zero. The loss function has some maxima and minima in the near field, but it increases monotonically in the Fresnel zone and in the far-field.

Manuscript received June 10, 1987.

The author is with Bilkent University, P.K.8 Maltepe, Ankara 06572, Turkey.

IEEE Log Number 8719379
On the other hand, the diffraction loss for the unequal size transducers facing each other cannot be found in the literature in a concise manner. This case is particularly important for the acoustic lens design as used in scanning acoustic microscopes [17] at relatively high frequencies. Typical acoustic lenses are made of a sapphire rod with a spherical lens cavity on one end and a circular transducer on the other. Acoustic attenuation losses in the liquid medium impose the condition that the radius of the acoustic lens be very small. If the size of the lens pupil is made small, only a fraction of incident acoustic energy will be useful for focusing. The resulting loss will be called diffraction loss. Moreover, reducing the size of the sapphire rod proportionately is not possible for practical reasons. The rods in length less than $1 \mathrm{~mm}$ are fragile and introduce serious handling problems. To keep the signal to noise ratio of the acoustic microscope system high, the diffraction loss in this long propagation distance must be minimized. The problem in this case is to find the optimum size of the transducer to give the minimum diffraction loss. It is possible to solve this problem by conventional methods [11]. Such a solution method would involve calculation of amplitude and phase of the field pattern at some distance and integrating it over the transducer extent to find the transducer output voltage. The calculation must be repeated for a number of different distances for optimization purposes. However, this method requires considerable amount of computer time and the results are with questionable accuracy. In this paper we will introduce a new and easy way of calculating the diffraction loss for such a problem. The results of the calculations are presented in a manner that will simplify the design procedure greatly. We will also introduce an approximate technique to calculate the diffraction loss in anisotropic materials.

\section{THEORY}

Using reciprocity theorem, Kino [18] and Auld [19] introduced a general scattering formula that relates the scattering coefficient at the electrical terminals of an acoustic transducer to acoustic field quantities around the flaw. Using this formula along with some approximations it is possible to obtain some closed form expressions. Subsequently, a backscattering formula was derived [20] for calculating the response of a transducer used in pulseecho mode facing a flaw. A similar formula can be easily 
derived for two tranducers facing each other as follows: We consider two transducers, one used as transmitter and the other as the receiver as shown in Fig. 1. The KinoAuld formula applies to this case directly. With the notation of Auld it can be stated as

$$
\delta \Gamma=(1 / 4 P) \oint_{S_{F}}\left(v_{1} \cdot T_{2}-v_{2} \cdot T_{1}\right) \cdot \hat{n} d S
$$

where $v$ is the particle velocity vector, $T$ is the stress tensor, $\hat{n}$ is the inward directed normal to the surface $S_{F}$ surrounding the scatterer. The field quantities with subscript 1 are obtained when the left-hand side (LHS) transducer is excited with incident power $P$ in the absence of scatterer and those with subscript 2 are obtained when the right-hand side (RHS) transducer is excited with the same power in the presence of the scatterer. $S_{F}$ is a closed surface surrounding the scatterer, $\delta \Gamma$ is the change of the electrical transmission coefficient due to the presence of scatterer.

We assume that the scatterer consists of a highly absorbing material with the same impedance as the propagation medium, therefore all the acoustic energy incident on it will be absorbed and nothing will be reflected. The scatterer is infinitely thin and planar. The coordinate system is selected such that $z=0$ plane is coincident with the scatterer. The surface $S_{F}$ is selected to be very close to the scatterer. For solution 1 (no scatterer, LHS transducer excited) we have the same field quantities on both sides of $S_{F}$ :

$$
\begin{aligned}
\text { LHS of } S_{F}: v_{1} & =v^{+} \\
T_{I} & =T^{+} \\
\text {RHS of } S_{F}: v_{l} & =v^{+} \\
T_{l} & =T^{+} .
\end{aligned}
$$

For solution 2 (with scatterer, RHS transducer excited) we have vanishing field quantities on the LHS of $S_{F}$ :

$$
\begin{aligned}
\text { LHS of } S_{F}: v_{2} & =0 \\
T_{2} & =0 \\
\text { RHS of } S_{F}: v_{2} & =v^{-} \\
T_{2} & =T^{-}
\end{aligned}
$$

where the superscripts show the direction of propagation of waves as well as the source of excitation. "+" and "-" superscripts refer to waves propagating in $+z$ and $-z$ direction, and excited by LHS and RHS transducers, respectively. Since $S_{F}$ is parallel to $z=0$ plane, $\hat{n}=a_{z}$ and $d S=d x d y$. Moveover, the transmission coefficient for solution 2 is zero. Therefore, (1) can be written as

$$
-\Gamma=(1 / 4 P) \oint_{S_{F}}\left(v \cdot{ }^{-} T^{+}-v \cdot{ }^{+} T^{-}\right) \cdot \hat{n} d S
$$

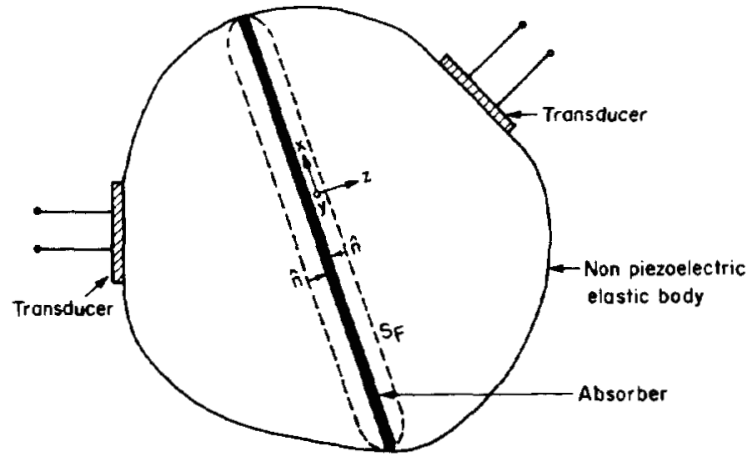

Fig. 1. Scattering geometry used in formulation.

where $\Gamma$ is the electrical transmission coefficient from one transducer to the other in the absence of scatterer. This equation can be written more explicitly in terms of components of vectors and tensors as

$$
\begin{aligned}
-\Gamma= & (1 / 4 P) \int_{-\infty}^{+\infty} \int_{-\infty}^{+\infty}\left(v_{x}^{-} T_{z x}^{+}+v_{y}^{-} T_{z y}^{+}+v_{z}^{-} T_{z z}^{+}\right. \\
& \left.-v_{x}^{+} T_{z x}^{-}-v_{y}^{+} T_{z y}^{-}-v_{z}^{+} T_{z z}^{-}\right) d x d y
\end{aligned}
$$

This equation is the same as $[20,(3)]$ with different interpretation of superscripts. Hence the rest of the derivation is the same. The result for a homogeneous and isotropic propagation medium is

$$
\begin{aligned}
& \Gamma=\left(1 / 8 \pi^{2} \omega P\right)\left\{c_{11} k_{0}^{2} \int_{-\infty}^{+\infty} \int_{-\infty}^{+\infty} k_{z} \Phi^{-}\left(k_{x}, k_{y}\right)\right. \\
& \cdot \Phi^{+}\left(-k_{x},-k_{y}\right) d k_{x} d k_{y} \\
& +c_{44} \int_{-\infty}^{+\infty} \int_{-\infty}^{+\infty} k_{z}^{\prime} \\
& \cdot\left[\Psi_{x}^{-}\left(k_{x}, k_{y}\right) \Psi_{y}^{-}\left(k_{x}, k_{y}\right) \Psi_{z}^{-}\left(k_{x}, k_{y}\right)\right] \\
& \cdot\left[\begin{array}{ccl}
k_{y}^{2}+k_{z}^{\prime 2} & -k_{x} k_{y} & k_{x} k_{z}^{\prime} \\
-k_{x} k_{y} & k_{x}^{2}+k_{z}^{\prime 2} & k_{y} k_{z}^{\prime} \\
k_{x} k_{z}^{\prime} & k_{y} k_{z}^{\prime} & k_{x}^{2}+k_{y}^{2}
\end{array}\right] \\
& \left.\cdot\left[\begin{array}{c}
\Psi_{x}^{+}\left(k_{x}, k_{y}\right) \\
\Psi_{y}^{+}\left(k_{x}, k_{y}\right) \\
\Psi_{z}^{+}\left(k_{x}, k_{y}\right)
\end{array}\right] d k_{x} d k_{y}\right\}
\end{aligned}
$$

where $c_{11}$ and $c_{44}$ are the elastic constants [21] of the isotropic medium; $\omega$ is the operating frequency; $P$ is the incident power; $\Phi$ and $\Psi$ are the Fourier transforms (hence angular spectra) of the scalar and vector potentials of the particle velocity field at $z=0$ plane. $k_{x}$ and $k_{y}$ are the wave vector components in $x$ and $y$ directions. $k_{z}$ and $k_{z}^{\prime}$ are written in terms of the wave numbers of longitudinal 
$\left(k_{0}\right)$ and shear waves $\left(k_{0}^{\prime}\right)$ as

$$
\begin{aligned}
& k_{z}=\left(k_{0}^{2}-k_{x}^{2}-k_{y}^{2}\right)^{1 / 2} \\
& k_{z}^{\prime}=\left(k_{0}^{\prime 2}-k_{x}^{2}-k_{y}^{2}\right)^{1 / 2} .
\end{aligned}
$$

Equation (4) expresses the transmission coefficient in terms of the angular spectra of scalar and vector potentials at a plane.

If we consider longitudinal waves only, (4) reduces to

$$
\begin{aligned}
\Gamma= & \left(c_{11} k_{0}^{2} / 8 \pi^{2} \omega P\right) \int_{-\infty}^{+\infty} \int_{-\infty}^{+\infty} k_{z} \Phi^{-}\left(k_{x}, k_{y}\right) \\
& \cdot \Phi^{+}\left(-k_{x},-k_{y}\right) d k_{x} d k_{y}
\end{aligned}
$$

where $\Phi^{+}$and $\Phi^{-}$are the angular spectra of the acoustic waves at $z=0$ plane generated by the LHS and RHS transducers, respectively. Equation (5) is a general formula that applies to two transducers. The $z=0$ plane could be selected at any convenient location. This expression can be reduced to that given by Johnson and Devaney [22] except for the constant factors.

\section{A. Diffraction Loss for Isotropic Case}

We can now return to the diffraction loss problem. Suppose that we have two longitudinal wave circular transducers of radii $a_{1}$ and $a_{2}$. The transducers are located coaxially and facing each other at a distance $d$. The coordinate axes are selected as shown in Fig. 2. Assuming piston type transducers the scalar potentials, $\phi_{1}$ and $\phi_{2}$, at the plane of each transducer can be written as

$$
\begin{aligned}
& \phi_{1}(0)=A_{1} \operatorname{circ}\left[\left(x^{2}+y^{2}\right)^{1 / 2} / a_{1}\right] \\
& \phi_{2}(d)=A_{2} \operatorname{circ}\left[\left(x^{2}+y^{2}\right)^{1 / 2} / a_{2}\right]
\end{aligned}
$$

where

$$
\operatorname{circ}(r)= \begin{cases}1 & r \leq 1 \\ 0 & r>0\end{cases}
$$

Since

$\Phi\left(k_{x}, k_{y}\right)=\iint_{-\infty}^{+\infty} \phi(x, y) \exp \left[-j\left(k_{x} x+k_{y} y\right)\right] d x d y$

the angular spectra at the same planes are [23]

$$
\begin{aligned}
& \Phi_{1}(0)=4 a_{1}^{2} A_{1} \text { jinc }\left[\left(a_{1} / \pi\right)\left(k_{x}^{2}+k_{y}^{2}\right)^{1 / 2}\right] \\
& \Phi_{2}(d)=4 a_{2}^{2} A_{2} \text { jinc }\left[\left(a_{2} / \pi\right)\left(k_{x}^{2}+k_{y}^{2}\right)^{1 / 2}\right]
\end{aligned}
$$

where jinc $(x)=J_{1}(\pi x) / 2 x$ and $J_{1}$ is the first order Bessel function of the first kind. To be able to apply (5) angular spectra must be specified at the same plane. Propagating angular spectrum from one plane to the other is taken care of by a simple exponential phase factor [23], [24]:

$$
\phi_{2}(0)=4 a_{2}^{2} A_{2} \text { jinc }\left[\left(a_{2} / \pi\right)\left(k_{x}^{2}+k_{y}^{2}\right)^{1 / 2}\right] \exp \left(-j k_{z} d\right) .
$$

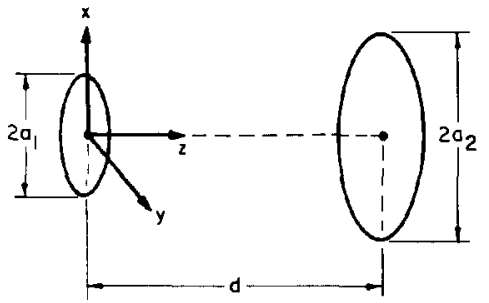

Fig. 2. Geometry and coordinate system for circular coaxial transducers separated by distance.

Applying (5) results in

$$
\begin{aligned}
\Gamma= & \frac{2 c_{11} k_{0}^{2} a_{1}^{2} a_{2}^{2} A_{1} A_{2}}{\pi^{2} \omega P} \int_{-\infty}^{+\infty} \int_{-\infty}^{+\infty} k_{z} \exp \left(-j k_{x} d\right) \\
& \cdot \text { jinc }\left[\left(a_{1} / \pi\right)\left(k_{x}^{2}+k_{y}^{2}\right)^{1 / 2}\right] \\
& \cdot \text { jinc }\left[\left(a_{2} / \pi\right)\left(k_{x}^{2}+k_{y}^{2}\right)^{1 / 2}\right] d k_{x} d k_{y}
\end{aligned}
$$

Due to the circular symmetry the double integral of (6) reduces to a single integral:

$$
\begin{aligned}
\Gamma= & \frac{4 c_{11} k_{0}^{2} a_{1}^{2} a_{2}^{2} A_{1} A_{2}}{\pi \omega P} \int_{0}^{k_{0}} k_{z}^{2} \exp \left(-j k_{z} d\right) \\
& \cdot \text { jinc }\left[\left(a_{1} / \pi\right)\left(k_{0}^{2}-k_{z}^{2}\right)^{1 / 2}\right] \\
& \cdot \text { jinc }\left[\left(a_{2} / \pi\right)\left(k_{0}^{2}-k_{z}^{2}\right)^{1 / 2}\right] d k_{z}
\end{aligned}
$$

where the evanescent fields are neglected. Equation (7) can be rewritten as an inverse Fourier transform operation:

$$
\begin{aligned}
\Gamma(d)= & \frac{4 c_{11} k_{0}^{2} a_{1}^{2} a_{2}^{2} A_{1} A_{2}}{\pi \omega P} F^{-1}\left\{k^{2} \text { jinc }\left(\frac{a_{1}}{\pi} \sqrt{k_{0}^{2}-k^{2}}\right)\right. \\
& \left.\cdot \operatorname{jinc}\left(\frac{a_{2}}{\pi} \sqrt{k_{0}^{2}-k^{2}}\right) \operatorname{rect}\left(k / k_{0}-1 / 2\right)\right\}\left.\right|_{x=d}
\end{aligned}
$$

where $F^{-1}$ is the inverse Fourier transform operator mapping from $k$-domain to $x$-domain, and

$$
\operatorname{rect}(k)=\left\{\begin{array}{ll}
1 & \text { if }|k|<1 / 2 \\
0 & \text { if }|k|>1 / 2
\end{array}\right. \text {. }
$$

If we assume transducers with zero conversion loss, we can relate the incident power and other parameters as

$$
P=c_{11} k_{0}^{3} a_{1}^{2} A_{1}^{2} / \omega=c_{11} k_{0}^{3} a_{2}^{2} A_{2}^{2} / \omega .
$$

Hence (8) reduces to

$$
\begin{aligned}
\Gamma(d)= & \frac{4 a_{1} a_{2}}{\pi k_{0}} \boldsymbol{F}^{-1}\left\{k^{2} \operatorname{jinc}\left(\frac{a_{1}}{\pi} \sqrt{k_{0}^{2}-k^{2}}\right)\right. \\
& \cdot \operatorname{jinc}\left(\frac{a_{2}}{\pi} \sqrt{k_{0}^{2}-k^{2}}\right) \\
& \left.\cdot \operatorname{rect}\left(k / k_{0}-1 / 2\right)\right\}\left.\right|_{x=d} .
\end{aligned}
$$


Equation (9) expresses the transmission coefficient as a function of distance between the two transducers in the form of a one-dimensional Fourier transformation. The diffraction loss, $L$, can be easily deduced from $L(d)=$ $-20 \log |\Gamma(d)|$.

\section{B. Diffraction Loss for Anisotropic Case}

Computing the propagation of bounded beams in anisotropic media is in general involved. For this purpose, angular spectrum method can be employed [25]. Along or close to pure mode directions approximate methods simplify the problem greatly. To include anisotropy we assume that $k_{r}$ is small and following Papadakis [11] we will replace $k_{0}$ by $k_{0}\left(1+b \theta^{2}\right)$ where $\theta$ is the deviation angle of the $k$ vector from $z$ axis and $b$ is the anisotropy factor as defined by Waterman. This approximation is valid even in the near field as long as $\theta^{4}$ term is negligible. For completeness we repeat the expressions for anisotropy parameter, $b$ for various crystals and orientations: $b=\left(c_{11}-\right.$ $\left.c_{12}-2 c_{44}\right)\left(c_{11}+c_{12}\right) /\left[2 c_{11}\left(c_{11}-c_{44}\right)\right]$ for cubic system [100] propagation; $b=2\left(2 c_{44}+c_{12}+c_{11}\right)\left(c_{11}+\right.$ $\left.2 c_{12}+c_{44}\right) /\left[3\left(c_{12}+c_{44}\right)\left(c_{11}+2 c_{12}+4 c_{44}\right)\right]$ for cubic system [111] propagation; $b=\left(c_{33}-c_{13}-2 c_{44}\right)\left(c_{33}+\right.$ $\left.c_{13}\right) /\left[2 c_{33}\left(c_{33}-c_{44}\right)\right]$ for hexagonal or tetragonal system $c$-axis, or trigonal system three-fold axis propagation.

Note that, for two-fold symmetry the $b \theta^{2}$ formulation above does not apply, even though it may be a pure mode direction. With $k_{r}^{2}=k_{x}^{2}+k_{y}^{2}$ and for $k_{r}$ small, we may write

$$
k_{r}^{2}+k_{z}^{2} \simeq k_{0}^{2}\left(1+b\left(k_{r} / k_{0}\right)^{2}\right)^{2}
$$

This equation can be rearranged to give approximately

$$
k_{r}^{2}(1-2 b)+k_{z}^{2} \simeq k_{0}^{2} \text {. }
$$

Hence

$$
k_{r}=\left(k_{0}^{2}-k_{z}^{2}\right)^{1 / 2} /(1-2 b)^{1 / 2} .
$$

Therefore (7) must be changed to

$$
\begin{aligned}
\Gamma \simeq \frac{4 a_{1} a_{2}}{\pi k_{0}} \int_{0}^{k_{0}} k_{z}^{2} \exp \left(-j k_{z} d\right) \\
\cdot \operatorname{jinc}\left(\frac{a_{1}}{\pi \sqrt{1-2 b}} \sqrt{k_{0}^{2}-k_{z}^{2}}\right) \\
\quad \cdot \operatorname{jinc}\left(\frac{a_{2}}{\pi \sqrt{1-2 b}} \sqrt{k_{0}^{2}-k_{z}^{2}}\right) d k_{z}
\end{aligned}
$$

It is seen that effect of anisotropy is the same as changing the dimensions of the transducers. In other words, the diffraction loss will be the same for the following two cases:

- Isotropic medium, circular transducers of size $a_{1}$ and $a_{2}$ separated by $d$.

- Anisotropic medium, circular transducers of size $a_{1} /(1-2 b)^{1 / 2}$ and $a_{2} /(1-2 b)^{1 / 2}$ separated by $d$.

One should note that above result is not valid in the near field if the $\theta^{4}$ term is no longer negligible.

\section{Pulsed Excitation}

The formula is valid for continuous wave signals, but it can be dependably used for pulsed systems where the pulse contains several cycles. For wide band systems, Fourier analysis can be easily used to find the pulse response due to diffraction. Suppose that the input pulse can be represented by a time function, $g(t)$. Suppose also that the frequency spectrum of this time function is $G(\omega)$ with $G(\omega)=F\{g(t)\}$. If the transducers can be assumed infinite bandwidth, the pulse obtained at the other transducer, $h(t)$, can be written as

$$
h(t)=\boldsymbol{F}^{-1}\{G(\omega) \Gamma(\omega)\}
$$

where $\Gamma(\omega)$ is the complex function that gives the transmission coefficient as a function of frequency.

\section{RESUlTS}

Evaluation of (9) requires the values of first order Bessel functions. Bessel function can be calculated accurately and relatively fast using recurrence relations [26]. The inverse Fourier transformation is done by the fast Fourier transform (FFT) algorithm [27]. Obviously, one must pay attention to aliasing effects during FFT operation. Because the function inside the brackets of (9) is nonzero only within an interval, the sampling on the function can be done to include the entire FFT input range. The number of points in the FFT must be large enough to reduce accuracy loss due to aliasing.

It is convenient to plot the diffraction loss as a function of normalized separation distance. A series of curves were obtained for various values of $a_{1}$ and $a_{2}$. It was seen that the curves corresponding to different values of $a_{1}$ are barely distinguishable from each other as long as $a_{2} / a_{1}$ ratio remains the same. This simplifies the presentation of results. One set of curves is applicable to almost all cases. Fig. 3 and Fig. 4 are the plots of the results. The horizontal axis is the distance between the transducers and it is normalized with respect to $a_{1}^{2} / \lambda$, where $\lambda$ is the wavelength in the propagation medium. The normalization of the vertical axis is achieved with respect to $a_{2} / a_{1}=1$ curve at $d=0$ where the diffraction loss is zero. At $d=$ 0 the calculated results for diffraction loss agree with the geometrical loss of $20 \log \left(a_{1} / a_{2}\right)$ for $a_{2}<a_{1}$, and 20 $\log \left(a_{2} / a_{1}\right)$ for $a_{1}<a_{2}$. The curves shown are for $a_{1}=$ $10 \lambda$ but they are nearly independent of the value of $a_{1}$ except when $a_{1}$ is very small. We have used a 8196 point FFT in calculating the given curves'. The program had to be written in FORTRAN 77 due to the presence of complex numbers. The calculations took about $3 \mathrm{~min}$ per curve on an IBM/XT computer with 8087 numerical coprocessor.

Since changing the frequency and changing the sepa-

\footnotetext{
'Numerical results presented in [20, Fig. 4] do not agree with our present results, due to an error in numerical calculations there. With the error corrected same results are obtained.
} 


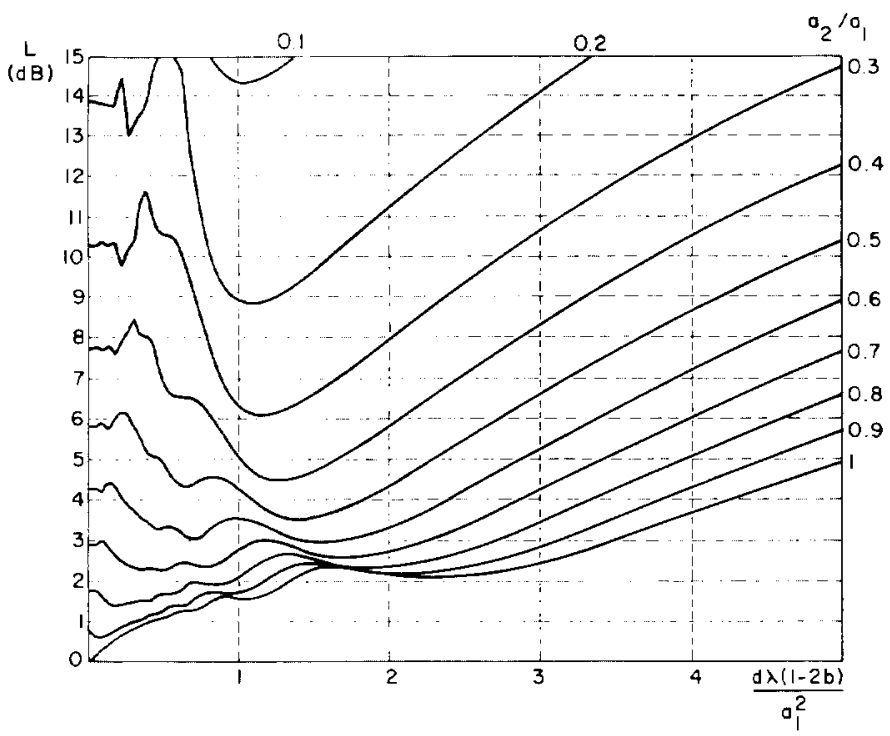

Fig. 3. Diffraction loss between circular transducers of radii $a_{1}$ and $a_{2}\left(a_{2}\right.$ $<a_{1}$ ) separated by $d$ in anisotropic medium with anisotropy parameter $b$.

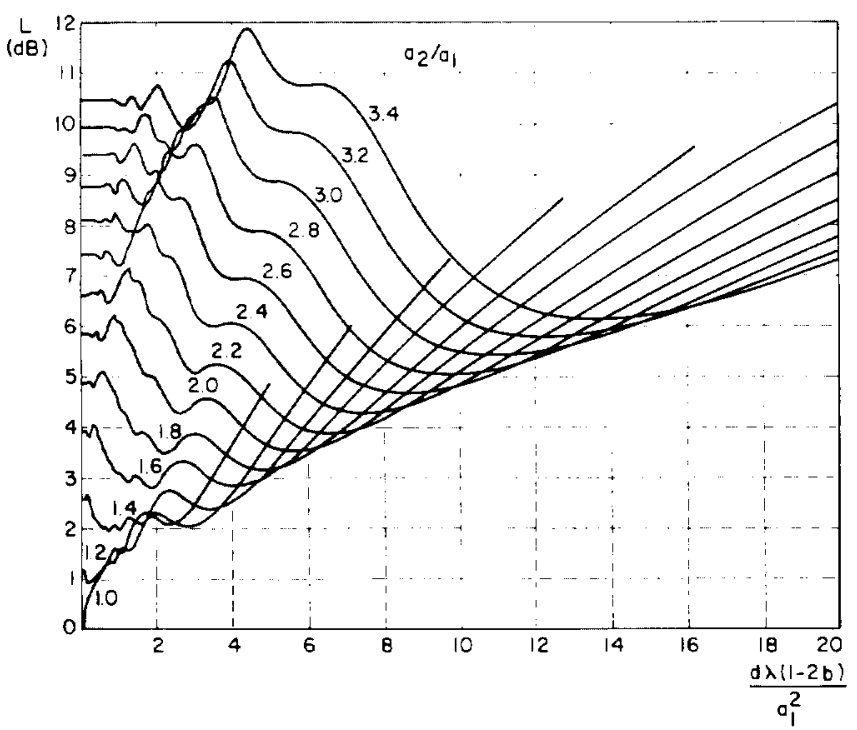

Fig. 4. Diffraction loss between circular transducers of radii $a_{1}$ and $a_{2}\left(a_{2}\right.$ $>a_{1}$ ) separated by $d$ in anisotropic medium with anisotropy parameter $b$.

ration are equivalent, the same set of curves can be used for pulse response determination. In this case horizontal axis of Figs. 3 and 4 becomes the inverse frequency axis, and curves shown become $\Gamma(1 / \omega)$ of $(12)$. One also needs the phase of the transmission coefficient for this purpose. Fig. 5 and Fig. 6 are the calculated excess phase curves. The phase curves start at zero and approach $\pi / 2$ asymptotically. They are pretty smooth in the far field meaning that there will be very small pulse distortion. In the near field and especially when the ratio of transducers is large, the phase curves loose the smoothness thus causing pulse distortion.

For the case $a_{1}=a_{2}$ the problem can be reduced to a single transducer geometry with a perfect and parallel re-

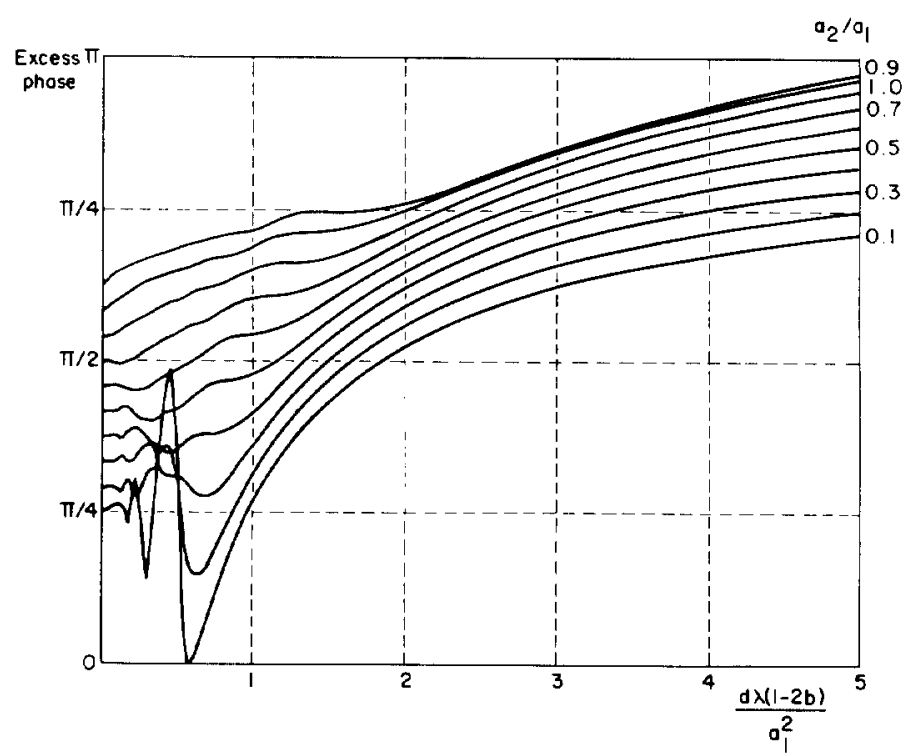

Fig. 5. Excess phase of transmission coefficient between circular transducers of radii $a_{1}$ and $a_{2}\left(a_{2}<a_{1}\right)$ separated by $d$ in anisotropic medium with anisotropy parameter $b$. Curves are displaced from each other vertically for clarity.

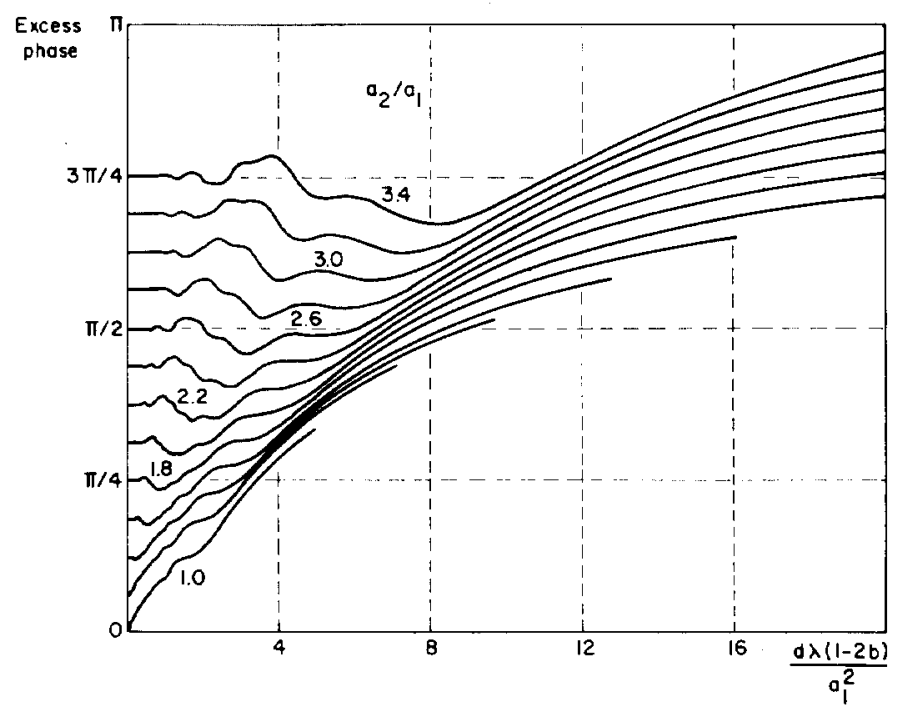

Fig. 6. Excess phase of transmission coefficient between circular transducers of radii $a_{7}$ and $a_{2}\left(a_{2}>a_{1}\right)$ separated by $d$ in anisotropic medium with anisotropic parameter $B$. Curves are displaced from each other vertically for clarity.

flector at a distance $d / 2$. For this case, given amplitude and phase curves are in good agreement with previous calculations and experimental results [11], [12].

For the anisotropic case the only thing that needs to be changed is the scaling of the horizontal axis. In this case horizontal axis label is changed to $d \lambda(1-2 b) / a_{1}^{2}$. Therefore, we may add one more case to the equivalent diffraction loss cases mentioned in the previous section: anisotropic medium, circular transducers of size $a_{1}$ and $a_{2}$ separated by $d /(1-2 b)$.

This result is in complete agreement with the results of 
Papadakis [11] as well as Cohen and Gordon [14] for the case $a_{1}=a_{2}$. If anisotropy factor $b$ is positive the separation between the transducers can be increased without changing the diffraction loss. On the other hand, if $b$ is negative the separation should be reduced to keep the same diffraction loss.

Inspection of Figs. 3 and 4 indicates that except when the values of $a_{1}$ and $a_{2}$ are very close to each other, the minimum diffraction loss is achieved when the two transducers are separated by a finite distance. This distance is slightly higher than the Fresnel distance $\left(a^{2} / \lambda(1-2 b)\right)$ of the larger transducer. This distance is at a point where the acoustic field produces a waist [1]. On the other hand, for $a_{2} / a_{1}=0.7$ the diffraction loss remains nearly the same at about $2.5 \mathrm{~dB}$ when $d$ is less than $2 a_{1}^{2} / \lambda(1-2 b)$. In all cases, when the separation distance gets very large, the diffraction loss increases by $6 \mathrm{~dB}$ per doubling of distance.

As an example of use of the plots consider an acoustic lens design at $2 \mathrm{GHz}$. The radius of the lens cavity is to be $40 \mu \mathrm{m}$ on one side of a $1 \mathrm{~mm}$ long sapphire rod. With $50^{\circ}$ half-opening angle the pupil radius becomes $30.6 \mu \mathrm{m}$. The problem is the determination of optimal transducer size for minimum diffraction loss. With $a_{1}=30.6 \mu \mathrm{m}, \lambda$ $=5.55 \mu \mathrm{m}, d=1000 \mu \mathrm{m}$ and $b=0.16$ (for sapphire [21]) we have $d \lambda(1-2 b) / a_{1}^{2}=4.03$. Inspection in Fig. 4 indicates that minimum diffraction loss is achieved when $a_{2} / a_{1}=1.4$, or $a_{2}=43 \mu \mathrm{m}$. For this case diffraction loss is about $2.5 \mathrm{~dB}$. The same problem in quartz $(\lambda=3.18$, $\left.b=-0.23, d \lambda(1-2 b) / a_{1}^{2}=4.97\right)$ results in a transducer radius of $49 \mu \mathrm{m}$ and a loss of $3 \mathrm{~dB}$. If one uses a 2 mm long sapphire rod, optimum transducer size and diffraction loss become $67 \mu \mathrm{m}$ and $4.2 \mathrm{~dB}$, respectively. In all cases, the total diffraction loss in the acoustic lens element will be twice the found values because of two-way propagation.

Note that in the acoustic lens design, minimization of diffraction loss may not be the only criterion. If uniform illumination of lens is desired for the purpose of higher resolution, one may deviate from the transducer sizes given above at the cost of higher diffraction loss.

\section{CONCLUSION}

We have presented a fast method of calculating diffraction loss between two transducers in isotropic or anisotropic media along or close to pure mode directions. Given graphs are in complete agreement with previous theoretical and experimental results for the limiting case of equal transducer sizes. They enable the determination of diffraction loss and excess phase between two coaxial circular transducers or of optimum transducer size for minimum diffraction loss. The same graphs can be used for predicting the pulse response. The theory developed is general: the transducer shape is not limited to circular geometry; one can easily adopt other transducer shapes. The plots given in this paper make the design of acoustic lenses to be used in scanning acoustic microscopes a simple task.

\section{REFERENCES}

[1] J. Zemanek. "Beam behavior within the nearfield of a vibrating piston," J. Acoust. Soc. Am., vol. 49, no. 1, pp. 181-191, 1971.

[2] J. C. Lockwood and J. G. Willette, "High-speed method of computing the exact solution for the pressure variations in the nearfield of a baffled piston," J. Acoust. Soc. Am., vol. 53, no. 3, pp. 735-741, 1973.

[3] P. R. Stephanishen, "Transient radiation from pistons in an infinite planar baffle," J. Acoust. Soc. Am., vol. 49, no. 5, pp. 1629-1638. 1971.

[4] W. L. Beaver, "Sonic nearfields of a pulsed piston radiator," $J$. Acoust. Soc. Am., vol. 56, no. 4, pp. 1043-1048, 1974.

[5] G. R. Harris, "Review of transient field theory for a baffled planar piston," J. Acoust. Soc. Am., vol. 70, no. 1, pp. 10-20, 1981

[6] J. N. Tjotta and S. Tjotta, "Nearfield and farfield of pulsed acoustic radiators," J. Acoust. Soc. Am., vol. 71, no. 4, pp. 824-834, 1982.

[7] D. Guyomar and J. Powers, "Transient fields radiated by curved surfaces-Application to focusing," J. Acoust. Soc. Am., vol. 76, no. 5, pp. 1564-1572, 1984

[8] W. L. Nyborg and R. B. Steele, "Nearfield of a piston source of ultrasound in an absorbing medium," J. Acoust. Soc. Am., vol. 78, pp. $1882-1891,1985$

[9] D. A. Hutchins, H. D. Mair, P. A. Puhach and A. J. Osei, "Continuous-wave pressure fields of ultrasonic transducers," J. Acoust. Soc. Am., vol. 80. no. 1, pp. 1-12, 1986.

[10] H. Seki, A. Granato, and R. Truell, "Diffraction effects in the ultrasonic field of a piston source and their importance in the accurate measurement of attenuation," J. Acoust. Soc. Am., vol. 28, no. 2 , pp. 230-238, 1956.

[11] E. P. Papadakis, "Ultrasonic diffraction loss and phase change in anisotropic materials," J. Acoust. Soc. Am., vol. 40, no. 4, pp. 863876,1966

[12] - "Ultrasonic phase velocity by the pulse-echo-overlap method incorporating diffraction phase correction," J. Acoust. Soc. Am., vol. 42, no. 5, pp. 1045-1051, 1967.

[13] - "Ultrasonic diffraction from single apertures with application to pulse measurements and crystal physics," in Physical Acoustics, W. P. Mason and R. N. Thurston, Eds. New York: Academic, 1979 , vol. 12, pp. $151-211$

[14] M. G. Cohen and E. L. Gordon, "Focusing of microwave acoustic beams," J. Appl. Phys., vol. 38, no. 5, pp. 2340-2344, 1967.

[15] M. B. Gitis and A. S. Khimunin, "Diffraction effects in ultrasonic measurements (Review),"Sov. Phys.-Acoust., vol. 14, no. 4, pp. $413-431,1969$.

[16] P. C. Waterman, "Orientation dependence of elastic waves in single crystals." Phys. Rev., vol. 113, no. 5, pp. 1240-1253, 1959.

[17] C. F. Quate, A. Atalar and H. K. Wickramasinghe, "Acoustic microscopy with mechanical scanning-A review," Proc. IEEE, 1979 , vol. 67 , pp. 1092-1114.

[18] G. S. Kino. "The application of reciprocity theory to scattering of acoustic waves by flaws," J. Appl. Phys., vol. 49, no. 6. pp. 31903199. 1978.

[19] B. A. Auld, "General electromechanical reciprocity relations applied to the calculation of elastic wave seattering coefficients, "Wave Mo tion, vol. 1, no. 1, pp. 3-10, 1979

[20] A. Atalar, "A backscattering formula for acoustic transducers," $J$. Appl. Phys., vol. 51, no. 6. pp. 3093-3098, 1980.

[21] B. A. Auld. Acoustic Fields and Waves in Solids, vol. 1. New York: John Wiley and Sons, 1973.

[22] R. K. Johnson and A. J. Devaney, "Transducer effects in acoustic scattering measurements," Appl. Phys. Lett., vol. 41, no. 7, pp. 622$624,1982$.

[23] J. Goodman, Introduction to Fourier Optics. New York: McGrawHill, 1968

[24] A. J. Devaney and G. C. Sherman, "Plane-wave representations for scalar wave fields," SIAM Rev., vol. 15, pp. 765-786, 1973.

[25] D. J. Vezzetti, "Propagation of bounded ultrasonic beams in anisotropic media," J. Acoust. Soc. Am., vol. 78, no. 3, pp. 1103-1108, 1985 .

[26] M. Abromowitz and I. A. Stegun, Handbook of Mathematical Functions. New York: Dover, 1970 , p. 385

[27] E. O. Brigham, The Fast Fourier Transform. Englewood Cliffs, N.J.: Prentice Hall, 1974. 


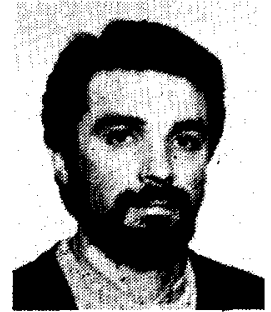

Abdullah Atalar was born in Gaziantep, Turkey, in 1954. He received the B.S. degree from Middle East Technical University, Ankara, Turkey in 1974, and the M.S. and Ph.D. degrees from Stanford University, Stanford, CA, in 1976 and 1978 , respectively, all in electrical engineering. His thesis work was on reflection acoustic microscopy.

From 1978 to 1980 he was first a Post Doctoral Fellow and later an Engineering Research Associate at Stanford University, he continued his work on acoustic microscopy. For eight months he was with Hewlett Packard Labs, Palo Alto, CA, engaged in photoacoustics re- search, In 1980 he joined the Middle East Technical University as an As sistant Professor. From 1982 to 1983 on leave from University, he was with Ernst Leitz Wetzlar, Wetzlar, West Germany, where he was involved in the development of the commercial acoustic microscope. He is presently an Associate Professor and chairman of the Electrical and Electronics Engineering Department at Bilkent University and is a part-time faculty member at Middle East Technical University. His current research interests include acoustic imaging, linear acoustics, and computer-aided design in Electrical Engineering.

Dr. Atalar is a member of Chamber of Electrical Engineers of Turkey In 1984 he was given the H. Tuğaç Foundation Award of TUBITAK, Turkey for his contributions to acoustic microscopy. 